COAGULATION OF GOLD HYDROSOLS BY ELECTROLYTES. 1563

\title{
CLXXII.-The Coagulation of Gold Hydrosols by Electrolytes. The Change in Colour, Influence of Temperature, and Reproducibility of the Hydrosol.
}

\author{
By Jĩanendra Nath Mukherjee and Basil Constantine \\ Papaconstantinou.
}

IN view of the results obtained by one of us (this vol., p. 350) with arsenious sulphide, it was thought desirable to investigate the influence of temperature on the precipitation of gold sols. In the course of this work it was found necessary to examine the reproducibility of the sols prepared by Zsigmondy's nucleus method. A spectrophotometric study of the changes in colour on coagulation has been made, and this has been utilised to measure the rate of precipitation.

\section{Preparation of the Hydrosol.}

All the sols were prepared by Zsigmondy's nucleus method. The specific conductivity of the water obtained from a pure tin condenser varied from $2 \times 10^{-6}$ to $3 \times 10^{-6}$ mhos. at $15^{\circ}$.

The nucleus solution was prepared as follows:

To 100 c.c. of pure water in a resistance-glass beaker were added 2 c.c. of a solution of chloroauric acid containing 6 grams of the acid in a litre, followed by 6 c.c. of a $N / 18$-solution of pure potassium carbonate. Five c.c. of a saturated solution of phosphorus in ether were diluted to 100 c.c. with pure ether, and the solution was added to the gold solution gradually-a few drops at a time. After each addition the solution was stirred, and this was continued until a deep chocolate colour was produced. It was then heated, and a "red" sol resulted.

To prepare the hydrosol proper, 2 c.c. of the chloroauric acid solution were added to 100 c.c. of water, followed by 6 c.c. of the potassium carbonate solution. The mixture was then heated to boiling, and 4 c.c. of the nucleus sol were added, followed by $4-5$ c.c. of a 0.03 per cent. solution of formaldehyde. The rubyred sol which was formed was boiled for a minute.

The hydrosols obtained in this way have all the properties of the best solutions prepared by Zsigmondy. In the cardioid ultramicroscope, the hydrosols show mostly green particles with a few brown ones. The sols contain 0.067 gram of gold per litre. 


\section{The Colour Changes in a Gold Hydrosol on the Addition of an Electrolyte.}

Zsigmondy (Annalen, 1898, 301, 46) studied the colour changes qualitatively only. In the numerous subsequent researches on the optical properties of gold sols, the change in the absorption on coagulation has not been examined.

The changes in colour were observed with a König-Martens spectrophotometer. The changes in the colour from red to blue on the addition of an electrolyte are mainly a result of a change in the absorption-coefficient of red and violet rays. The change is greatest in the red region. There is a limiting value of the absorption-coefficient corresponding with the blue colour of the sol, and these limiting values are independent of the nature of the electrolytes used. The coefficients were calculated from the equation

$$
K=K_{1} / 2 \cdot 306=\frac{1}{d} \log _{10} \frac{\tan ^{2} \alpha}{\tan ^{2} \alpha_{0}} *
$$

(Hildebrand, Zeitsch. Elektrochem., 1908, 14, 349).

In studying the precipitation, equal volumes of sol and electrolyte were mixed. The coefficients for the pure sol refer to that for the sol diluted with an equal volume of pure water. The electrolytes used were potassium chloride, sodium chloride, barium chloride, potassium nitrate, strontium nitrate, and potassium sulphate.

The results are given in Fig. 1, and are the mean of observations with different samples of sols and different electrolytes. The wave-lengths are correct within $\pm 1 \mu \mu$. It is interesting to note that in the region near $523 \mu \mu$ there is scarcely any change in the absorption-coefficient.

It will be seen later that the results obtained for the absorption of light by the hydrosols, prepared under exactly similar conditions, differ a little among themselves except in this region, which is close to the spectral region, where the absorption is at a maximum (near $506 \mu \mu$ ) (compare Ehrenhaft, Ann. Physik, 1903, [iv], 11, 489).

Assuming the particles in a gold sol to be spherical and that there are many particles to a wave-length of light, Garnett (Phil. Trans., 1904, $[A]$, 203, 385; 1906, $[A]$, 205, 237) deduces that the maximum of absorption should be for light of wave-length $533 \mu \mu$. Mie (Ann. Physik, 1908, [iv], 25, 377) also deduces from his theory that the maximum

* $a_{0}=$ the angle for water or electrolyte solution alone. 
of the pure (corrected) absorption lies in the region 525 to $550 \mu \mu$. He further shows that for spherical particles the region of maximum of absorption does not vary with the size of the particles, although the value of the absorption-coefficient depends on the size. According to these theories, the colour of a gold sol by trans-

FIG. 1.

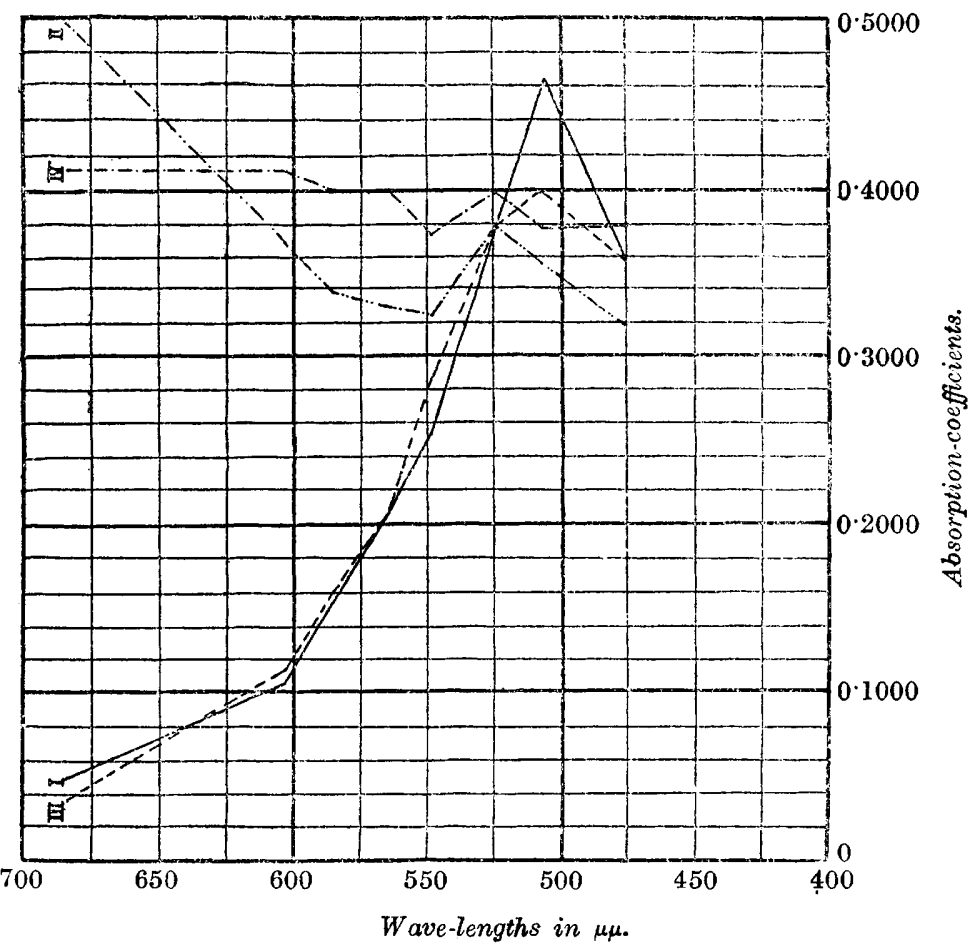

I. Hydrosol diluted with water.

II. ", mixed with electrolyte.

III. Nucleus sol dilute with water.

IV. Hydrosol mixed with electrolyte.

mitted light is the result of two properties of the gold particles or aggregates :

(1) Minute, spherical gold particles have a fairly well-defined maximum of absorption in the green. Reflection in the ordinary sense is very weak in this case, and the colour is due to the absorption of the green light, which is the colour of the light scattered most strongly by these particles.

(2) With increasing aggregation, as the size becomes comparable 


\section{MUKHERJEE AND PAPACONSTANTINOU: THE COAGULATION}

to the wave-length of light there is increasing reflection. When the size becomes sufficiently large, the optical discontinuity of the medium becomes manifest as a turbidity. As is to be expected from the properties of metallic gold, red and yellow rays are mostly reflected, and the transmitted light becomes correspondingly weaker in the red and yellow.

The observed constancy of the absorption-coefficient therefore indicates two possibilities, namely: (1) The reflection of green light is relatively small for gold $(47 \cdot 3$ per cent. for $500 \mu \mu)$. The minute particles in a gold sol scatter green light almost completely, and the part played by reflection is negligible. The formation of clusters, therefore, may be taken not to affect the absorption.

(2) It is possible that the change in scattering is counterbalanced by that due to reflection with the progress of aggregation. Garnett remarks that "when the particles are not sufficiently thickly distributed to satisfy the condition of there being many particles to a wave-length of light . . . the absorption that we have investigated is therefore not present" (Phil. Trans., 1904, $[A], 203$, 402). Indeed, it is found that, on coagulation, the intensity of the blue light $(500 \mu \mu)$ diminishes for the sol. This is in accordance with the theories mentioned, as neither of them leads to the expectation of a constant absorption on aggregation. The theoretical aspect is further complicated by the fact that the shape of the aggregate has to be considered.

However, the observed constancy is striking, as it coincides with the region of maximum absorption deduced by Garnett and Mie from the properties of metallic gold.

It is possible that the scattering of green light is a characteristic property of the gold atom, and is independent of cluster formation.

\section{The Measurement of the Rate of Coagulation with the Spectrophotometer.}

The change in absorption on coagulation is great in the red region, and it has been utilised to measure the rate of precipitation at the ordinary temperature. The reciprocal of the time required to reach the limit of absorption is a measure of the rate of coagulation. As a characteristic change in the sol itself is utilised, this method has an advantage over others dependent on an arbitrarily selected stage of change. For the fine sols used in this work, the direct method of determining the rate of decrease in the number of particles is not possible with ordinary ultramicroscopes (Zsigmondy, Zeitsch. physikal. Chom., 1918, 92, 600).

This simple spectrophotometric method can be used with a suit- 
able ultramicroscope with economy of labour and time. The number of particles corresponding with the absorption-coefficient values can be determined by the ultramicroscope, and their mutual relationship can be utilised to substitute the spectrophotometer for the tedious ultramicroscopic work. This is possible for the whole range of changes in colour from red to blue, but is not applicable when the limit of absorption has been reached.

The influence of the concentration of an electrolyte has been followed with the spectrophotometer for potassium chloride, potassium sulphate, potassium nitrate, and barium chloride. The mean results of three observations are given in tables I-III. The absorption-coefficients are for light of wave-length $683 \mu \mu$. The observations show that for low concentrations of an electrolyte the "limiting" values of absorption are not reached, and coagulation practically stops at a certain stage.

TABLE I.

Electrolyte: Potassium Chloride.

Time in minutes after mixing equal volumes of electrolyte. and sol.

$\overline{0 \cdot 5}$
$1 \cdot$
$1 \cdot 5$
2
3
5
9
13
15

Absorption-coefficients for various concentrations.

$\begin{array}{lll}N / 24 . & N / 26 . & N / 28 . \\ 0.0453 & 0.0453 & 0.0453 \\ 0.3732 & 0.2867 & 0.1683 \\ 0.438 & 0.3630 & 0.2257 \\ 0.4497 & 0.4046 & - \\ - & 0.438 & 0.2777 \\ - & 0.4497 & 0.3431 \\ - & - & 0.3836 \\ - & - & 0.4263 \\ - & - & 0.438 \\ & - & 0.4497\end{array}$

TABLE II.

Potassium Nitrate.

Concentration.

$\begin{array}{ccll}\text { Time. } & N / 24 . & N / 26 . & N / 30 \\ - & 0.0453 & 0.0453 & 0.0453 \\ 0.5 & 0.3336 & - & - \\ 1 & 0.4263 & 0.2866 & 0 \cdot 269 \\ 1.5 & 0.4497 & 0.3271 & 0.3143 \\ 2 & - & 0.3629 & 0.3336 \\ 3 & - & 0.4156 & 0 \cdot 3732 \\ 4 & - & 0.438 & 0.394 \\ 5 & - & 0.4497 & -\overline{4263} \\ 8 & - & - & 0.438 \\ 10 & - & - & 0.4497 \\ 16 & - & - & \end{array}$


TABLE III.

Barium Chloride.

\begin{tabular}{cccc}
\multicolumn{4}{c}{ Concentration. } \\
Time. & $0.852 N / 900$. & $0 \cdot 852 N / 1000$. & $0.852 N / 1100$. \\
-1 & $0 \cdot 0453$ & $0 \cdot 0453$ & $0 \cdot 0453$ \\
1 & $0 \cdot 2257$ & - & $0 \cdot 1603$ \\
2 & $0 \cdot 2867$ & - & $0 \cdot 2007$ \\
4 & $0 \cdot 3529$ & - & $0 \cdot 2687$ \\
5 & $0 \cdot 3836$ & $0 \cdot 3051$ & $0 \cdot 3051$ \\
7 & $0 \cdot 438$ & 0.3431 & $0 \cdot 3237$ \\
8 & 0.4497 & - & $0 \cdot 3336$ \\
9 & 0.4497 & 0.3836 & $0 \cdot 3529$ \\
11 & - & $0 \cdot 4263$ & $0 \cdot 363$ \\
13 & - & 0.4497 & $0 \cdot 363$ \\
16 & - & - & 0.3732
\end{tabular}

These experiments were carried out within a short interval and with the same sol. There is scarcely any difference between the coagulating effects of the three potassium salts. Smoluchowski (Zeitsch. physikal. Chem., 1917, 92, 129) has deduced the following equation for the rate of decrease in the total number of particles:

$$
n_{t}=\frac{n}{1+\frac{t}{T}} \cdot . \quad \cdot \quad \cdot \quad \cdot \quad \cdot
$$

where $n$ is the total number of particles in unit volume just after mixing the sol with the electrolyte (zero time), $n_{t}$ is the number at time $t$ (second), and $T$ is characteristic of the rate of coagulation.

Zsigmondy finds that when the minimum coagulation-time has been reached, two particles, on coming in contact as a result of their Brownian movement, are held together by forces of cohesion. The work of Westgren and Reitstötter (Zeitsch. physikal. Chem., $1918,92,750$ ) lends support to this, as all these authors find that the radius of the effective sphere of attraction (as defined by Smoluchowski) is nearly equal to twice the radius of the particles. Under these conditions,

$$
T=\frac{3}{4} \frac{N_{0} \eta}{R \theta n_{\epsilon}} \quad \cdot \quad \cdot \quad \cdot \quad \cdot \cdot \cdot
$$

where $\eta=$ the viscosity of the solution.

$R=$ the gas constant.

$N_{0}=$ the Avogadro number.

$\theta=$ the absolute temperature.

$\epsilon=$ the fraction of mutual collisions between the particles which result in a stable union. 
For a constant value of $n_{t}=n^{\prime}$, it is evident that

or

$$
\begin{aligned}
& \epsilon . t=\text { constant . . . . . . } \\
& \epsilon=\frac{k}{t}
\end{aligned}
$$

The reciprocal of the times for a definite change is thus directly proportional to the fraction of collisions which result in coalescence, and hence measures the rate of coagulation. The data obtained on the influence of concentration will be discussed on another occasion. The equations given above will be discussed later when dealing with the influence of temperature.

\section{The Influence of Temperature.}

The method used by one of us (loc. cit.) was adopted. The great variation in the colour of a ruby-red gold sol makes it suitable to use a definite shade of violet-red or bluish-violet for comparison. Standards for comparison were made by arresting the colour-change at a selected stage with gelatin, and, with care, perfectly reproducible standards can be prepared. The times necessary for the sol to change to the colour of the standard are given below.

TABLE IV.

Electrolyte: $0.852 \mathrm{~N} / 1000$-Barium Chloride.

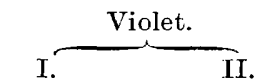

Sol. A ..... 6 min. 6 min.

Sol. B ..... 2 min. $2 \mathrm{~min} .25 \mathrm{sec}$.

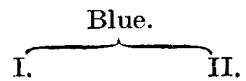

29 min. $\quad 29$ min. $10 \mathrm{~min} .45 \mathrm{sec} .10 \mathrm{~min} .30 \mathrm{sec}$.

The results are the mean of six observations taken separately by each of us. Sol B was obtained by boiling sol A for a few minutes and then cooling. It will be seen that the boiling produces a change in the sol.

The concentration of the electrolyte used for producing the change in the colour should be such as will bring about a slow rate of precipitation. About 2 c.c. of a 2 per cent. gelatin solution (liquid) were added to 10 c.c. of the sol-electrolyte mixture. The same standards remain satisfactory for ten to twelve days. Wide test-tubes were used to secure a suitable depth of colour. As is well known, ruby-red gold sols are extremely sensitive to impurities. Reproducible results can only be obtained if the vessels are cleaned with sufficient care. The glass vessels were washed with conductivity water after the usual cleaning with hot chromic acid and distilled water. The test-tubes were washed with boiling dis- 
tilled water (after chromic acid had been used), then with conductivity water, and finally by passing steam derived from conductivity water. They were then dried in a steam-oven. There is a noticeable difference between cleaning with distilled water and conductivity water. Table $\mathrm{V}$ illustrates the reproducibility of the results.

TABLE V.

N/1000-Strontium Nitrate.

\begin{tabular}{|c|c|c|c|c|c|c|c|c|c|c|c|c|}
\hline & & & & & & Obs & vation & & & & & \\
\hline & & & & & & 3. & 4 & & & . & & 3. \\
\hline mes. & Min. & Sec. & Min. & Sec. & Min. & Sec. & Min. & Sec. & Min. & Sec. & Min. & Sec. \\
\hline t. & 1 & 10 & 1 & 6 & 1 & 11 & 1 & 13 & 1 & 0 & 1 & 12 \\
\hline .. & 5 & 45 & 5 & 48 & 5 & 30 & 5 & 30 & 3 & 30 & 6 & 0 \\
\hline
\end{tabular}

In each case, three to five readings were taken. With all these precautions, at times discordant results were obtained, which were probably due to the occasional presence of particles of dust. The agreement between the different observations and the appearance of the characteristic blue colour is the surest indication of the absence of impurities. With long intervals, it is difficult to avoid dust. A slow change in colour is also much less easily perceptible to the eye. For these reasons, it was found suitable to use concentrations of electrolytes which change the colour to blue within an hour. As different standards were used, they are indicated as $V_{1}, V_{2}$, etc., for violet standards, and in a similar manner for blue standards.

TABLE VI.

\begin{tabular}{|c|c|c|c|c|}
\hline \multirow{2}{*}{$\begin{array}{l}\text { Stan- } \\
\text { dards. }\end{array}$} & & \multicolumn{3}{|c|}{ Temperatures. } \\
\hline & & $15^{\circ}$. & $30^{\circ}$. & $50^{\circ}$. \\
\hline$V$ & Sol. C. & $5 \mathrm{~min}$. & $10 \mathrm{~min}$. & $8 \mathrm{~min} .30 \mathrm{sec}$. \\
\hline & Sol. D. & $\begin{array}{l}30 \text { sec. } \\
42 \text { sec. }\end{array}$ & $\begin{array}{l}10 \mathrm{sec} . \\
18 \mathrm{sec}\end{array}$ & $\begin{array}{l}10 \mathrm{sec} . \\
12 \mathrm{sec} .\end{array}$ \\
\hline
\end{tabular}

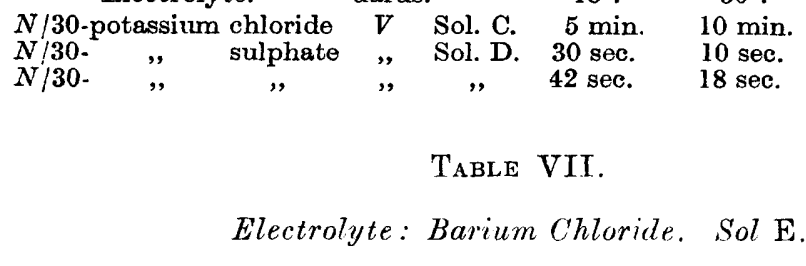

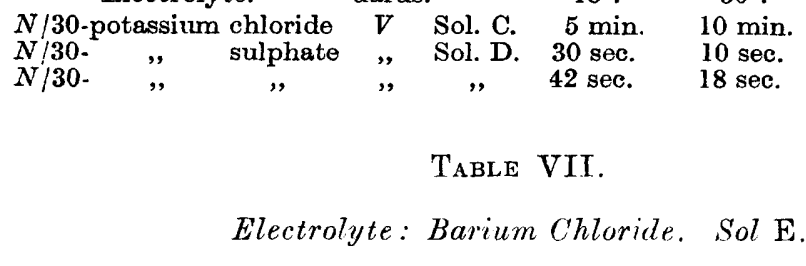

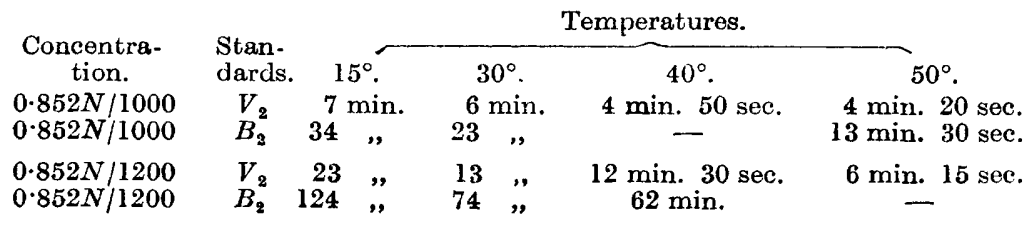


OF GOLD HYDROSOLS BY ELECTROLYTES.

TABLE VIII.

Electrolyte: Strontium Nitrate. Sol F.

\begin{tabular}{|c|c|c|c|c|}
\hline & & & Temperature. & \\
\hline $\begin{array}{c}\text { Concentration. } \\
N / 1000 \ldots \ldots \ldots \ldots \ldots \ldots \ldots \\
N / 1000 \ldots \ldots \ldots \ldots \ldots \ldots \ldots \ldots\end{array}$ & $\begin{array}{c}\text { Standards. } \\
V_{3} \\
B_{3}\end{array}$ & $\begin{array}{l}15^{\circ} . \\
8 \text { min. } 10 \text { sec. } \\
8 \text { min. } 15 \text { sec. }\end{array}$ & $\begin{array}{c}30^{\circ} . \\
20 \text { sec. } \\
1 \text { min. } 40 \text { sec. }\end{array}$ & $\begin{array}{l}50^{\circ} . \\
8 \text { sec. } \\
45 \text { sec. }\end{array}$ \\
\hline
\end{tabular}

With barium chloride and strontium nitrate there is a distinct increase in the rate of coagulation with rise of temperature. Potassium chloride has been examined at different concentrations. Temperature has a relatively small effect in the case of the potassium salts. With potassium chloride, both an increase and a decrease in the coagulation times have been observed with rise of temperature. This behaviour is similar to that observed by one of us in the case of arsenious sulphide sols and aluminium sulphate. The effect of temperature is also dependent on the concentration of the electrolyte.

Westgren (Arkiv. Kem. Min. Geol., 1918, 7, No. 6) has found that, in the case of coarse gold sols, sodium chloride and hydrochloric acid show an increase in the rate of precipitation with rise in temperature. With sodium hydroxide, he found no change in the rate with rise of temperature. From equations (1) and (2), if $n$ and $n^{\prime}$ have the same values, we have

$$
\frac{t \theta \epsilon}{\eta}=k, \text { a constant . . . . . . }
$$

where

$$
k=\frac{3}{4} \frac{n-n^{\prime}}{n \cdot n^{\prime}} \cdot \frac{N_{0}}{R} \text {. }
$$

From equation (5), the variation in $t$ can be calculated. If $\epsilon$ remains constant, we have

$$
\frac{t \theta}{\eta}=\text { constant . . . . . . . . . }
$$

that is, the times are proportional to the values of $\frac{\eta}{\theta}$ at different temperatures. At $15^{\circ}, 30^{\circ}, 40^{\circ}$, and $50^{\circ}, \frac{\eta}{\theta}$ has the values $3.96 \times 10^{-5}, 3.3 \times 10^{-5}, 2.1 \times 10^{-5}$, and $1.7 \times 10^{-5}$ respectively.

The results with hydrosols of gold and arsenious sulphide show that $\epsilon$ varies with temperature, and that the variation is determined by the nature of the electrolyte. For any given concentration of an electrolyte, $\epsilon$ measures its coagulating power. On the adsorption theory, the coagulating power of an ion is determined by its adsorbability. In order to explain the results on the 
adsorption theory, it must be assumed, as pointed out previously, that the adsorbability of an ion depends on the temperature (this vol., p. 350).

\section{The Reproducibility of the Hydrosols prepared by the Nucleus Method.}

In the course of this investigation, it was noticed that these sols, even when prepared under identical conditions, do not give

FIG. 2.

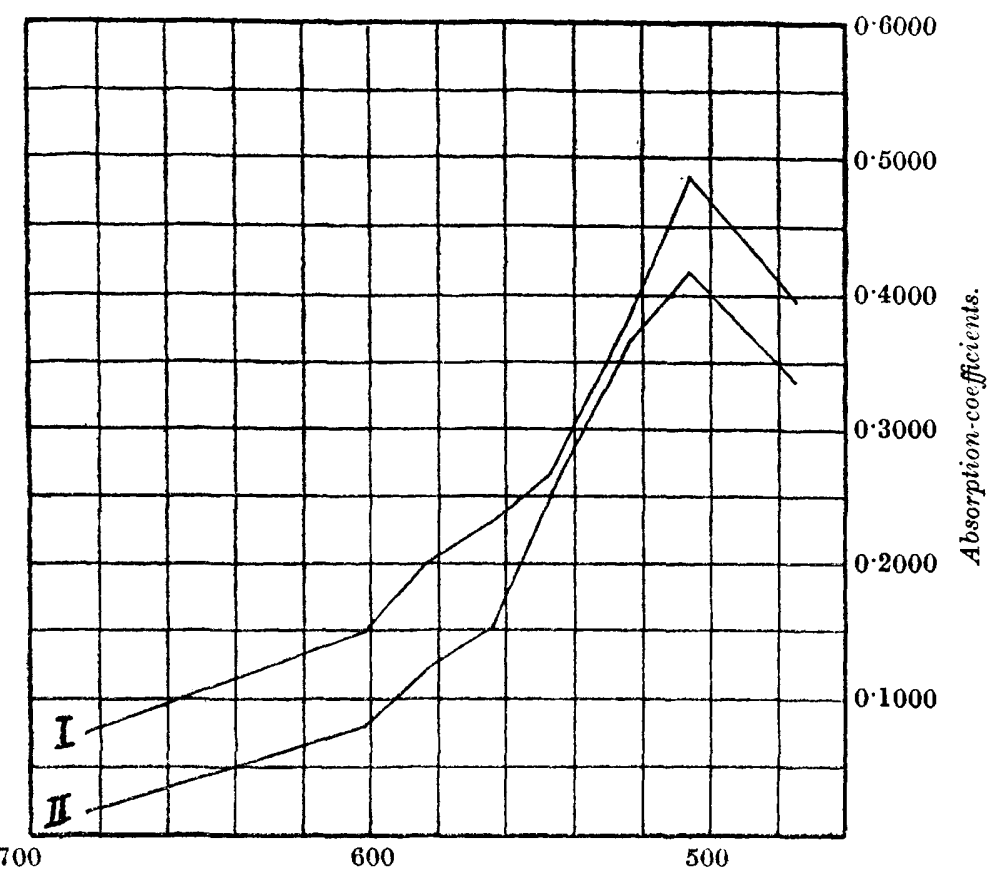

Wave-lengths in $\mu \mu$.

I. Upper limit of absorption.

II. Lower limit of absorption.

the same coagulation times. According to Zsigmondy, the sols are reproducible if the quality of the water is unchanged. We find that the various samples of gold sols (prepared in an identical manner) show small, but perceptible, differences in coagulation times, although they have constant gold numbers. This is due to the fact that the gold numbers are independent of the slight differences in the quality of the sols. 
Some twelve samples were prepared under the same conditions, and the absorption and the coagulation times were compared. The latter are extremely sensitive to any change in the sol. The limits of variation in the absorption-coefficients will be evident from Fig. 2 .

It will be observed that the variation is least in the region 520 to $540 \mu \mu$.

A sol, on keeping, undergoes somewhat irregular changes, which may in part be due to particles of dust getting in accidentally, and in part to the fungus that grows in these sols. For this reason, it is necessary to vary one factor only at a time and compare its effects. Table IX illustrates the variations, the same "violet" and "blue" standards being used. The times given are the mean of three to five observations. The last column gives the time that has passed since the preparation of the sol. The sols were kept in resistance-glass vessels. Sol I is an "old" preparation kept for two months. It was boiled twice during this interval to prevent organic growths.

\section{TABle IX.}

Electrolyte: $0.852 \mathrm{~N} / 1200-$ Barium Chloride.

\begin{tabular}{|c|c|c|c|c|c|c|c|c|c|}
\hline \multirow{2}{*}{\multicolumn{2}{|c|}{ Standards. }} & \multicolumn{2}{|c|}{ Sol G. } & \multicolumn{2}{|c|}{ Sol H. } & & & \multicolumn{2}{|c|}{ Sol I. } \\
\hline & & $\underset{\mathbf{3}}{\operatorname{Min} .}$ & $\begin{array}{r}\text { Sec. } \\
15\end{array}$ & $\begin{array}{r}\text { Min. } \\
\mathbf{3}\end{array}$ & $\begin{array}{r}\text { Sec. } \\
35\end{array}$ & & $\begin{array}{l}\text { ge. } \\
\text { lays }\end{array}$ & Min. & $\mathbf{S}$ \\
\hline , & ...................... & 2 & 30 & 8 & 0 & 3 & ,, & 11 & 0 \\
\hline ", & & 1 & 4 & 6 & 15 & 4 & ", & 12 & 30 \\
\hline ," & 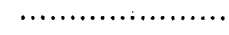 & 2 & 0 & 6 & 52 & 5 & , & 12 & 30 \\
\hline Blü & ... & 2 & 45 & 4 & 10 & 6 & , & 12 & 30 \\
\hline Blue.. & 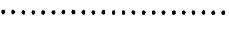 & 7 & 0 & 6 & 30 & 2 & ", & & \\
\hline ".. & $\ldots \ldots \ldots \ldots \ldots \ldots \ldots \ldots \ldots$ & 5 & 45 & 18 & $\mathbf{0}$ & 3 & ," & 22 & 0 \\
\hline .. & $\ldots \ldots \ldots \ldots \ldots \ldots \ldots \ldots$ & 4 & 40 & 19 & 0 & 4 & ," & 30 & 0 \\
\hline , $\quad$. & $\ldots \ldots \ldots \ldots \ldots \ldots \ldots \ldots$ & 9 & 0 & 22 & 30 & $\mathbf{5}$ & , & 30 & 30 \\
\hline . & & 9 & 0 & 14 & 0 & 6 & ", & 33 & 0 \\
\hline
\end{tabular}

On the other hand, reproducible results were obtained in some instances. At the suggestion of Professor Donnan, the gold number of a number of soaps has been determined. The sols give a constant gold number for the same soap solution.

We desire to express our thanks to Professor F. G. Donnan, F.R.S., for his kind interest in this work, and also to Dr. J. C. Ghosh.

Chemical Laboratory, UnIVEtsity Colteas, London. [Received, October 12th, 1920.] 